\title{
On the Observability of Bearing-only SLAM
}

\author{
Teresa Vidal-Calleja, Mitch Bryson, Salah Sukkarieh, \\ Alberto Sanfeliu and Juan Andrade-Cetto
}

\begin{abstract}
In this paper we present an observability analysis for a mobile robot performing SLAM with a single monocular camera. The aim is to get a better understanding of the well known intuitive behavior of these systems, such as the need for triangulation to features from different positions in order to get accurate relative pose estimates. The characterisation of the unobservable directions is made using the nullspace basis of the stripped observability matrix. This allow us to identify which vehicle motions are required to maximise the number of observable states in the system, which in turn affects accuracy in the estimation process. The analysis is performed by modelling the system in the continuous time domain as piecewise constant. Simulation results using an extended information filter are shown to verify the results of the observability analysis.
\end{abstract}

\section{INTRODUCTION}

Bearing-only SLAM refers to the case in which only the angle to a feature with respect to the sensor is measured and where no range information is available. Monocular vision is one such case in which a single measurement step can only provide incomplete information for the reconstruction of the state space, with the consequence that feature locations cannot be estimated from a single image, and must be computed from the tracking of features over multiple views. From a systems theory viewpoint, we can say that one measurement step in bearing-only SLAM renders the system only partially observable.

That one step bearing-only SLAM is unobservable, and that it can only become observable by integrating measurements at different time steps once the appropriate motions are made, are known facts within the SLAM community. One of the aims in this communication is to provide a formal explanation to issues that are intuitively known or that have been commonly addressed with geometrical concepts such as the motion parallax. For example, we show with our observability analysis why nothing can be said about the location of features that are sensed by a bearing-only sensor that has no translational motion, or that repeated measurements to a feature along the line of sight of a forward moving camera do not aid in recovering its range. By providing a formal explanation to these simple issues, we pave the road to understanding more complicated behaviors particular of bearing-only SLAM systems; for instance, a constant velocity model for a free moving camera in $6 \mathrm{D}$

Teresa Vidal-Calleja, Juan Andrade-Cetto and Alberto Sanfeliu are with the Institut de Robòtica i Informàtica Industrial, CSIC-UPC. Llorens Artigas 4-6, Barcelona 08028, Spain. tvidal, cetto, sanfeliuliri.upc.edu

Mitch Bryson and Salah Sukkarieh are with ARC Centre of Excellence in Autonomous Systems,ACFR, University of Sydney. NSW 2006, Australia. m.bryson, salah@cas.edu.au requires the integration of measurements of at least three different time steps in order to guarantee observability.

The performance of the estimator in a bearing-only SLAM system is strongly related to the trajectories described by the sensor. Ultimately, one would want to drive the system such as to avoid unobservable states; only then we can guarantee bounded estimation uncertainty. The analysis presented here will uncover the circumstances that help a bearing-only SLAM system recover from unobservable conditions.

Related to our work, in [1] the authors study the observability of a mobile robot localising itself in a $2 \mathrm{D}$ world using bearing-only observations to known features. In [2] the authors study the observability of multiple robots attempting to localise themselves w.r.t the position of other robots. In both of these papers a non-linear observability analysis is performed over the equations in each system using the technique in [3] which involves computing the Lie derivatives over the non-linear equations of the system. In [4], a nonlinear observability analysis for the planar robot absolute localisation problem is performed. Their work concludes that absolute localisation is observable only after a certain number of anchors (markers) have been observed. An observability analysis for the nonlinear systems typical of SLAM models could also be performed by numerically evaluating the observability Gramians.

Instead of performing a nonlinear observability analysis, an alternative explored in this work is to consider our nonlinear system in its error form which allows the system to be approximated with piecewise constant linear functions. This allows us to use tools in linear systems observability analysis [5] which greatly reduce the complexity of the analysis. These tools also allow us to derive explicit expressions of the unobservable directions in the state space that give insight as to what actions should be made, or what features should be seen so as to improve observability of the entire estimation process. Moreover, by characterising explicitly the form of the unobservable subspaces we get a better understanding of the behavior of the system.

Following the technique in [5], we analyse here a synchrodrive planar mobile robot performing SLAM using the measurement modality of bearing-only vision. The approach consists in determining the number of segments needed to obtain a full rank of the so-called Stripped Observability Matrix. A similar methodology was applied to study the observability of SLAM using inertial sensors for a UAV in [6] and [7]. 


\section{ObSERVABILITy OF LinEAR Systems}

Observability is a notion that plays a major role in filtering and reconstruction of states from inputs and outputs. The observability condition is an indicator whether or not the system contains all the necessary information to perform the estimation with an error which is bounded. In particular, for the case of SLAM, observability implies a bounded error both in the localisation of the vehicle and the features.

Formally, observability simply asks if an initial state $\mathbf{x}(0)$ can be uniquely deduced from the history of observations. This requires that the Observability Gramian

$$
\mathcal{O}(0, t) \triangleq \int_{0}^{t} e^{\mathbf{F}^{\top} \tau} \mathbf{H} \mathbf{H}^{\top} e^{\mathbf{F} \tau} d \tau
$$

be non singular, or equivalently that the nullspace of $\mathcal{O}$ is $\mathbf{0} \in \mathbb{R}^{n}$, where $\mathbf{F}$ and $\mathbf{H}$ are the plant and measurement matrices, and $n$ is the size of the state. If this condition is met, then all states are observable.

For time invariant systems, the observability condition can be reduced to check whether the matrix

$$
\mathcal{Q} \triangleq\left[\begin{array}{c}
\mathbf{H} \\
\mathbf{H F} \\
\vdots \\
\mathbf{H F}^{n-1}
\end{array}\right]
$$

has rank $n$ or not.

A time variant system can be approximated by a piecewise constant system with little loss of accuracy and with no loss of the characteristic behavior of the system [5]. For a piecewise constant system, its $k$-th segment dynamic model is

$$
\begin{aligned}
\dot{\mathbf{x}}(t) & =\mathbf{F}(t) \mathbf{x}(t)+\mathbf{G}(t) \mathbf{u}(t) \\
\mathbf{z}(t) & =\mathbf{H}(t) \mathbf{x}(t),
\end{aligned}
$$

where,

$$
\begin{aligned}
& \mathbf{F}(t)=\mathbf{F}_{1}, \mathbf{H}(t)=\mathbf{H}_{1} \quad t_{0}<t<t_{1} \\
& \mathbf{F}(t)=\mathbf{F}_{2}, \mathbf{H}(t)=\mathbf{H}_{2} \quad t_{1}<t<t_{2} \\
& \mathbf{F}(t)=\mathbf{F}_{k}, \mathbf{H}(t)=\mathbf{H}_{k} \quad t_{k-1}<t<t_{k}
\end{aligned}
$$

The observability matrix for each segment is

$$
\mathcal{Q}_{k}=\left[\begin{array}{c}
\mathbf{H}_{k} \\
\mathbf{H}_{k} \mathbf{F}_{k} \\
\vdots \\
\mathbf{H}_{k} \mathbf{F}_{k}^{n-1}
\end{array}\right] .
$$

The system is instantaneously observable at time $k$ if its nullspace is equal to $\mathbf{0}$. However, to guarantee observability of a piecewise constant system, the full-rank condition must hold for the Total Observability Matrix (TOM)

$$
\mathcal{Q}_{\mathrm{TOM}, k}=\left[\begin{array}{c}
\mathcal{Q}_{1} \\
\mathcal{Q}_{2} e^{\mathbf{F}_{1} \tau_{1}} \\
\vdots \\
\mathcal{Q}_{k} e^{\mathbf{F}_{k-1} \tau_{k-1}} \cdots e^{\mathbf{F}_{1} \tau_{1}}
\end{array}\right]
$$

with $\tau_{k}$, the time interval between $t_{k}$ and $t_{k-1}$.

The Stripped Observability Matrix (SOM), a simplified form of the TOM is defined as

$$
\mathcal{Q}_{\mathrm{soM}, k}=\left[\begin{array}{c}
\mathcal{Q}_{1} \\
\mathcal{Q}_{2} \\
\vdots \\
\mathcal{Q}_{k}
\end{array}\right]
$$

and it can be shown [5] that if

$$
\mathcal{N}\left(\mathcal{Q}_{k}\right) \subset \mathcal{N}\left(\mathbf{F}_{k}\right), \quad \forall k
$$

then

$$
\mathcal{N}\left(\mathcal{Q}_{\text {SOM }}\right)=\mathcal{N}\left(\mathcal{Q}_{\text {TOM }}\right)
$$

So, when the condition holds, the analysis is as simple as calculating the SOM instead of the TOM. Using the SOM for the study of the observability of piecewise constant systems is much simpler than using the TOM, since the exponentials $e^{\mathbf{F}_{k} \tau_{k}}$ need not be computed.

The systems studied in this paper trivially hold this condition.

\section{Planar Vehicle SLAM}

A planar vehicle is usually controlled by linear and angular velocities $v$ and $\omega$, respectively. The process model used to predict the position and orientation of the centre of projection of the camera on such a model is given by

$$
\left[\begin{array}{c}
\dot{x} \\
\dot{y} \\
\dot{\theta}
\end{array}\right]=\left[\begin{array}{c}
v \cos \theta \\
v \sin \theta \\
\omega
\end{array}\right] .
$$

We assume a measurement model that makes bearing observations to point features only. In the most simple planar case, the bearing to a feature is

$$
\varphi=\tan ^{-1}\left(\frac{r_{y}}{r_{x}}\right)-\theta,
$$

where $\mathbf{r}=\mathbf{m}-\mathbf{p}$ is the $2 \mathrm{~d}$ distance vector between any given feature and the sensor, and $\theta$ is the sensor orientation.

The following table contains some of the notation used throughout the rest of the article.

$$
\begin{array}{ll}
\mathbf{p}=[x, y]^{\top} & \begin{array}{l}
\text { sensor position } \\
\text { orientation angle of the vehicle }
\end{array} \\
v & \text { sensor linear velocity } \\
\omega & \text { sensor angular velocity } \\
\mathbf{m}=\left[m_{x}, m_{y}\right]^{\top} & \text { feature location } \\
\mathbf{r}=\left[r_{x}, r_{y}\right]^{\top} & \text { relative position between a feature and the sensor } \\
\varphi & \text { bearing angle to a feature measured by the sensor }
\end{array}
$$

This model, as most other SLAM models, is nonlinear. We can express the SLAM system in its indirect (error) form where the state contains the vehicle position error $\tilde{\mathbf{p}}$, the vehicle orientation error $\tilde{\theta}$, and the feature position errors $\tilde{\mathbf{m}}^{(1)}, \ldots, \tilde{\mathbf{m}}^{(i)}$. The state error is defined as the difference between the true state and the estimated state. 


\section{A. World-centric}

The state transition for the $k$-th piecewise segment of this indirect form is

$$
\left[\begin{array}{c}
\dot{\tilde{x}} \\
\dot{\tilde{y}} \\
\dot{\tilde{\theta}} \\
\dot{\tilde{m}}_{x}^{(1)} \\
\dot{\tilde{m}}_{y}^{(1)} \\
\vdots \\
\dot{\tilde{m}}_{x}^{(i)} \\
\dot{\tilde{m}}_{y}^{(i)}
\end{array}\right]=\mathbf{F}_{k}\left[\begin{array}{c}
\tilde{x} \\
\tilde{y} \\
\tilde{\theta} \\
\tilde{m}_{x}^{(1)} \\
\tilde{m}_{y}^{(1)} \\
\vdots \\
\tilde{m}_{x}^{(i)} \\
\tilde{m}_{y}^{(i)}
\end{array}\right] .
$$

Considering only one feature, we have five states in the state vector (three vehicle pose states and 2 feature position states). The state transition matrix for the $k$-th segment of the continuous piecewise linear system is:

$$
\mathbf{F}_{k}=\left[\begin{array}{ccccc}
0 & 0 & -v_{k} \sin \theta_{k} & 0 & 0 \\
0 & 0 & v_{k} \cos \theta_{k} & 0 & 0 \\
0 & 0 & 0 & 0 & 0 \\
0 & 0 & 0 & 0 & 0 \\
0 & 0 & 0 & 0 & 0
\end{array}\right] .
$$

The measurement model for the $k$-th piecewise segment, expressed also in this indirect form, is $\tilde{\mathbf{z}}=\mathbf{H}_{k} \tilde{\mathbf{x}}$, where

$$
\mathbf{H}_{k}=\left[\begin{array}{lllll}
\frac{r_{y}}{d^{2}} & -\frac{r_{x}}{d^{2}} & -1 & \frac{-r_{y}}{d^{2}} & \frac{r_{x}}{d^{2}}
\end{array}\right]
$$

and $d^{2}=r_{x}^{2}+r_{y}^{2}$. Note also, that although not explicitly indicated in the notation, the relative feature position $\mathbf{r}$, and the distance $d$ in $\mathbf{H}_{k}$ are also with respect to the $k$-th piecewise segment.

Now, let us consider the first time segment. The observability matrix ${ }^{1}$, from (6) is

$$
\mathcal{Q}_{1}=\left[\begin{array}{ccccc}
\frac{r_{y}}{d^{2}} & -\frac{r_{x}}{d^{2}} & -1 & -\frac{r_{y}}{d^{2}} & \frac{r_{x}}{d^{2}} \\
0 & 0 & -\frac{r_{y}}{d^{2}} v \sin \theta-\frac{r_{x}}{d^{2}} v \cos \theta & 0 & 0
\end{array}\right] .
$$

The resulting nullspace basis for this instantaneous observability matrix is,

$$
\left\{\left[\begin{array}{c}
r_{x} \\
r_{y} \\
0 \\
0 \\
0
\end{array}\right],\left[\begin{array}{l}
1 \\
0 \\
0 \\
1 \\
0
\end{array}\right],\left[\begin{array}{c}
-r_{x} \\
0 \\
0 \\
0 \\
r_{y}
\end{array}\right]\right\} .
$$

Thus the unobservable modes are $r_{x} \tilde{x}+r_{y} \tilde{y}, \tilde{x}+\tilde{m}_{x}$, and $-r_{x} \tilde{x}+r_{y} \tilde{m}_{y}$. An important conclusion from (15) is that all of the nullspace basis vectors are orthogonal to the vehicle orientation state (i.e. the third element of every nullspace basis vector is zero). This means that the vehicle orientation is the only completely observable state from the original state vector; except for the case of $v=0$, in which case the null space basis is of dimension 4 , and the orientation

\footnotetext{
${ }^{1}$ In (14) and in the rest of the observability matrices shown throughout the paper, zero rows are not shown, as they do not contribute to determining the unobservable directions of the state space. These typically come from evaluating the powers of the transition matrix when constructing them.
}

is also unobservable. Note also that the values of $r_{x}$ and $r_{y}$ are irrelevant, that is, regardless on where the feature is located with respect to the sensor, there will always be three unobservable modes in one time segment (except of course, for the singular case of a feature on the optical centre of the camera $r_{y}=0$ ).

Extending the analysis for two time segments the SOM takes the form in (8) evaluated for $k=2$, and computing its nullspace basis we get

$$
\left\{\left[\begin{array}{l}
1 \\
0 \\
0 \\
1 \\
0
\end{array}\right],\left[\begin{array}{l}
0 \\
1 \\
0 \\
0 \\
1
\end{array}\right]\right\}
$$

Adding more features or time segments does not modify the observability conditions. That is, the system will remain partially observable with non-observable modes $\tilde{p}_{x}+$ $\sum_{j} \tilde{m}_{x}^{(j)}$ and $\tilde{p}_{y}+\sum_{j} \tilde{m}_{y}^{(j)}$ as the nullspace basis above shows for the case of only one feature. Needless to say, the nullspace basis for the TOM is exactly the same.

\section{B. World-centric with Anchors}

Other authors have analysed the conditions of observability using anchors instead of relative mapping for the planar case of range and bearing [8]. In that case, only one time segment is needed. For the planar bearing-only case analysed here, one anchor and two time segments are needed for the system to become fully observable.

The state space remains the same as the one used in subsection III-A. The position of at least one feature in the environment is known and our measurement model is augmented to localise the positions of other features w.r.t the known feature(s). The known feature(s) are referred as anchors (Andrade and Sanfeliu [8]) or markers (Bicchi et al. [4]).

The new measurement matrix for the $k$-th segment is,

$$
\mathbf{H}_{k}=\left[\begin{array}{ccccc}
\frac{r_{y}}{d^{2}} & -\frac{r_{x}}{d^{2}} & -1 & -\frac{r_{y}}{d^{2}} & \frac{r_{x}}{d^{2}} \\
\frac{a_{y}^{(1)}-y}{\left(d a^{(1)}\right)^{2}} & -\frac{a_{x}^{(1)}-x}{\left(d a^{(1)}\right)^{2}} & -1 & 0 & 0 \\
& & \ddots & & \\
\frac{a_{y}^{(j)}-y}{\left(d a^{(j)}\right)^{2}} & -\frac{a_{x}^{(j)}-x}{\left(d a^{(j)}\right)^{2}} & -1 & 0 & 0
\end{array}\right],
$$

where $d a=\left(a_{y}-y\right)^{2}+\left(a_{x}-x\right)^{2}$ and $j$ is the number of anchors.

Notice that more measurements of the relative position of the vehicle are available because of the anchor, therefore the instantaneous observability matrix is larger than in the case of world-centric localisation with no anchors.

Performing the same analysis as in the above subsection, the nullspace basis of the instantaneous observability matrix using the world-centric model with one anchor and one 
unknown feature is,

$$
\left\{\left[\begin{array}{c}
0 \\
0 \\
0 \\
-r_{x, 1} \\
-r_{y, 1}
\end{array}\right],\left[\begin{array}{c}
-a_{x}^{(1)}+x_{1} \\
-a_{y}^{(1)}+y_{1} \\
0 \\
\frac{r_{x, 1} a_{y}^{(1)}-r_{y, 1} a_{x}^{(1)}-m_{x, 1} y_{1}+m_{y_{1} x_{1}}}{\left(-m_{y, 1}+y_{1}\right)\left(-a_{y}^{(1)}+y_{1}\right)} \\
0
\end{array}\right]\right\} \text {. }
$$

Adding one or more anchors reduces the dimension of the nullspace basis of the instantaneous observability matrix to only one vector,

$$
\left\{\left[\begin{array}{c}
0 \\
0 \\
0 \\
-r_{x, 1} \\
-r_{y, 1}
\end{array}\right]\right\} \text {. }
$$

Moreover, performing the analysis for two time segments, either with one or more anchors, renders the SOM fully observable.

\section{Sensor-centric}

Alternatively, let us consider the state as the relative position error of the vehicle w.r.t the features rather than separate global vehicle and map feature errors. In other words, let us consider a sensor-centric model, where $\tilde{\mathbf{r}}=\tilde{\mathbf{m}}-\tilde{\mathbf{p}}$. The state vector becomes

$$
\mathbf{x}=\left[\begin{array}{c}
\tilde{\mathbf{r}}^{(1)} \\
\tilde{\mathbf{r}}^{(2)} \\
\vdots \\
\tilde{\mathbf{r}}^{(i)} \\
\tilde{\theta}
\end{array}\right]
$$

The dimension of the state vector is reduced by two, and the nullspace basis of $\mathcal{Q}_{1}$ is reduced to one vector for each feature, i.e.

$$
\left\{\left[\begin{array}{c}
r_{x, 1}^{(1)} \\
r_{y, 1}^{(1)} \\
0 \\
0 \\
\vdots \\
0 \\
0 \\
0
\end{array}\right],\left[\begin{array}{c}
0 \\
0 \\
r_{x, 1}^{(2)} \\
r_{y, 1}^{(2)} \\
\vdots \\
0 \\
0 \\
0
\end{array}\right], \cdots,\left[\begin{array}{c}
0 \\
0 \\
0 \\
0 \\
\vdots \\
r_{x, 1}^{(i)} \\
r_{y, 1}^{(i)} \\
0
\end{array}\right]\right\}
$$

In the case of having only one feature, the nullspace rank of the instantaneous observability matrix is one, and again, the observable state is the orientation. As the number of features increases, the orientation state remains the only observable state in the instantaneous observability matrix.

For the particular case of having a feature aligned exactly in front of the camera, $\left(r_{y}=0\right)$, the unobservable mode for the one time segment observability matrix is $\tilde{r}_{x}$. This means that having only such measurement available, the relative position between the vehicle and the feature is unrecoverable, but the orientation can be recovered. A similar situation happens when the feature is aligned with the image plane, $\left(r_{x}=0\right)$, and the unobservable mode is $\tilde{r}_{y}$. Although it is unlikely that such case will be detected by the sensor, unless an omnidirectional camera is used.

In contrast to global localisation or world-centric model with no anchors, this SLAM system becomes fully observable in two time-segments, as can be verified from the SOM and the TOM becoming full-rank. This result is of special importance, as it gives a theoretical grounding to what has become common practice in SLAM systems. That is, on the use of relative maps as opposed to world-centric maps.

As in the one time segment case, the system will remain unobservable when a feature is aligned with the field of view of the sensor, $r_{y}=0$, with an unobservable mode $\tilde{r}_{x}$. The same does not happen however for a feature aligned along the $y$ axis, as the difference in $r_{x}$ will move away from zero during the second segment, and the SOM and TOM will become full rank.

When there is no translation (pure rotation), the nullspace basis augments by one vector. Therefore, neither the position nor the orientation can be fully recovered (only a combination of the two). In this case, the SOM and TOM are not full rank, and consequently there is no way to estimate the whole state for bearing-only SLAM when $v=0$. On the other hand, when there is no rotation, the system is completely observable in two time segments, except again, for the unfortunate case when a feature is aligned along the line of sight of the sensor.

\section{RESUlts}

In this section we show simulation results which demonstrate the way in which the observability affects the information in the estimation process of SLAM. An extended information filter [9] is used to fuse encoder data and bearingonly observations using the process and measurement models in (9) and (10) and using a world-centric state vector as shown in (11). The information filter maintains the inverse of the covariance matrix (the information matrix) associated with and extended Kalman filter implementation. The rank of the information matrix at any point in the estimation has a direct link to the rank of the observability matrix of the system. A rank deficient information matrix indicates that the information along a given axis of the state space is zero and this state is unrecoverable due to partial observability in the system.

The information matrix allows us to represent complete initial uncertainty in the estimated states as zero information rather than infinite covariance, and provides a better estimator for representing in the initial infinite uncertainty of the position of a feature along the line of sight of a single observation. The only remaining issue with the extended information filter for bearing-only SLAM is that the Jacobian of the observation model cannot be evaluated until a sufficiently accurate estimate of the range to the feature is derived from subsequent observations of a feature from different poses. To overcome this, the Jacobians of the process and measurement models are evaluated using the simulated truth data instead. This assumption is obviously not applicable in 


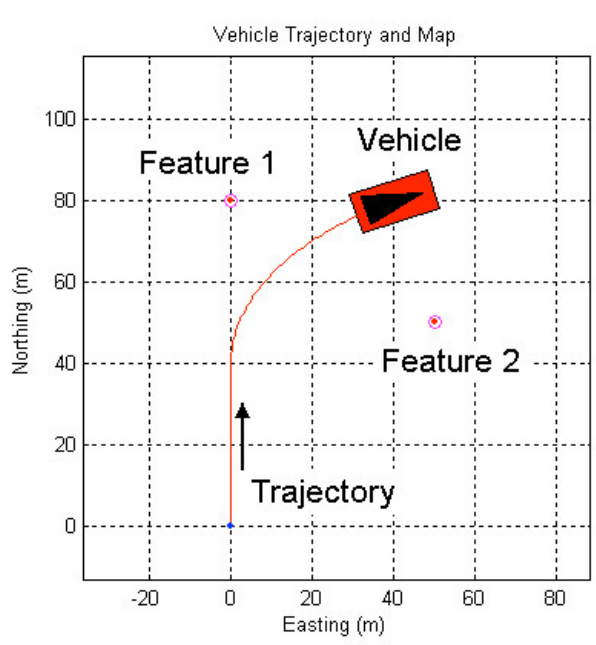

Fig. 1. Vehicle trajectory and feature map for the simulation. The vehicle is stationary for 10 seconds then moves northerly for 4 seconds and turns and moves to the north-east for another five seconds. There are two features in the map, visible to the vehicle for the entire duration of the simulation.

a real scenario. However, it does not affect the value of the inverse covariance matrix in the information filter. We can therefore examine the value of the inverse covariance matrix as an indication of the errors in the estimation process, under the assumption that linearisation errors in the extended information filter are ignored.

Figure 1 illustrates the trajectory taken by the vehicle. The vehicle starts off facing north and is stationary for 10 seconds. There are two observable features, one located directly north of the vehicle and one located to the north east. Both features are within the observation range of the onboard feature sensor for the entire duration of the simulation. After 10 seconds the vehicle moves north with a velocity of $10 \mathrm{~m} / \mathrm{s}$ for 4 seconds and then turns and continues to the east without stopping for another 6 seconds. Table 1 shows the parameters used in the simulation.

TABLE I

Simulation/Information Filter Parameters

\begin{tabular}{|c|c|c|}
\hline $\begin{array}{c}\text { Simulation } \\
\text { Time Step }\end{array}$ & $\begin{array}{c}\text { Prediction } \\
\text { Time Step }\end{array}$ & $\begin{array}{c}\text { Observation } \\
\text { Time Step }\end{array}$ \\
\hline $0.1 \mathrm{secs}$ & $0.1 \mathrm{secs}$ & $0.1 \mathrm{secs}$ \\
\hline $\begin{array}{c}\text { Velocity } \\
\text { Process Noise }\end{array}$ & $\begin{array}{c}\text { Turn Rate } \\
\text { Process Noise }\end{array}$ & $\begin{array}{c}\text { Observation } \\
\text { Noise }\end{array}$ \\
\hline $0.5 \mathrm{~m} / \mathrm{s}$ & $0.05 \mathrm{rad} / \mathrm{s}$ & $0.01 \mathrm{rads}$ \\
\hline
\end{tabular}

Figure 2 shows the rank of the information matrix during the simulation. Since there are two observed features, visible for the entire simulation, there are seven states in the state vector in total. The system model in this case corresponds to that in (11). While the vehicle is stationary, only two states are observable corresponding to a combination of the vehicle and feature position and vehicle orientation angle. As the vehicle moves north after 10 seconds, the vehicle's non-zero velocity and the multiple observations of feature 2 from different pose angles result in a rank increase of

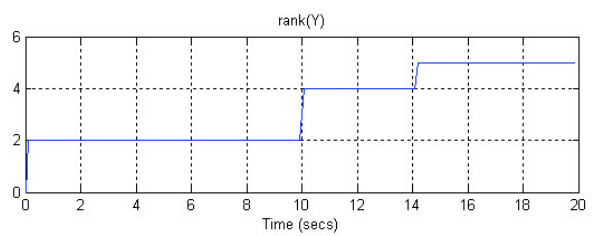

Fig. 2. Rank of the information matrix. After 14 seconds the information matrix is rank 5 and has a rank deficiency of 2 , representing the globally unobservable mode specified in Equation 16.

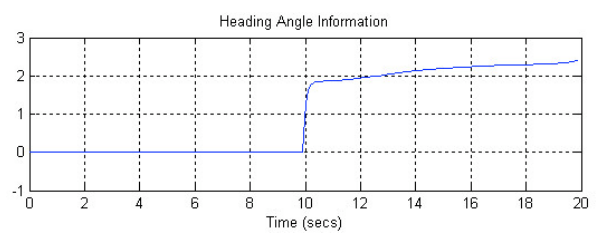

Fig. 3. Marginalised orientation angle information. While the vehicle is stationary, the orientation angle is unobservable and thus has zero information. When the vehicle begins to move at the 10 second mark, the orientation angle becomes observable and thus the information increases.

two in the information matrix corresponding to an increase in the number of observable states. The orientation angle is observable and also the relative position of the second feature to the vehicle and the relative east position of the first feature to the second feature but not the relative north position of the first feature due to the vehicle moving along the line of sight to this feature. When the vehicle moves to the northeast after 14 seconds, the rank of information matrix rises to five corresponding to the relative north position of feature 1 w.r.t the vehicle becoming observable. The remaining rank deficiency of two corresponds to the unobservable modes in (16).

Figure 3 shows the marginalised vehicle orientation angle information $^{2}$ during the simulation. The information about the orientation angle starts at zero and only begins to increase when the orientation angle becomes observable as the vehicle begins to move forward at the 10 second mark.

Figure 4(a) shows the marginalised information from the information matrix corresponding to the modes $\tilde{p}_{x}+\sum_{j} \tilde{m}_{x}^{(j)}$ and $\tilde{p}_{y}+\sum_{j} \tilde{m}_{y}^{(j)}$. These are the unobservable modes shown in (16) which cannot be made observable through any number of time segments or any amount of platform maneuvering. The information corresponding to these states thus remains at zero throughout the entire simulation.

Figures 4(b) and 4(c) show the marginalised information corresponding to the state space modes $\tilde{p}_{x}-\tilde{m}_{1 x}$ and $\tilde{p}_{y}-\tilde{m}_{1 y}$ (i.e. the relative position of the vehicle w.r.t feature 1) and $\tilde{m}_{1 x}-\tilde{m}_{2 x}$ and $\tilde{m}_{1 y}-\tilde{m}_{2 y}$ (i.e. the relative position of feature 1 w.r.t feature 2). All information about the relative position of features w.r.t the vehicle or to other features is zero (they are unobservable) before the vehicle starts moving. After the 10 second mark when the vehicle starts moving

\footnotetext{
${ }^{2}$ Marginalisation of a single state from a multi-dimensional inverse covariance (information) matrix involves computing the Schur complement of the diagonal matrix element of the state of interest (see [10] for details).
} 

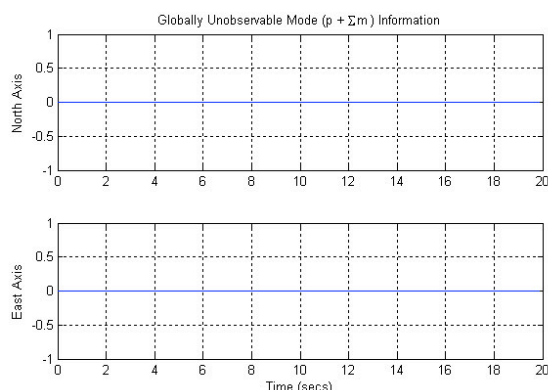

(a) Modes $\tilde{p}_{x}+\sum_{j} \tilde{m}_{x}^{(j)}$ and $\tilde{p}_{y}+\sum_{j} \tilde{m}_{y}^{(j)}$.
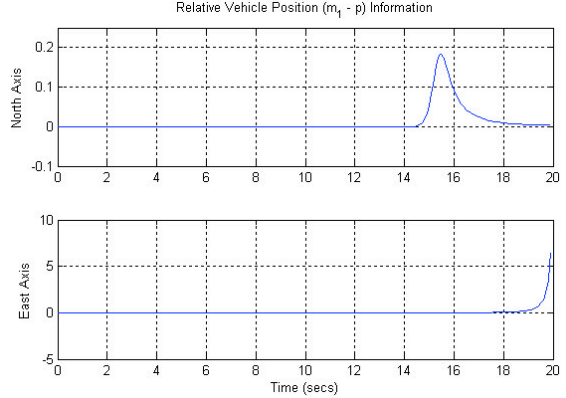

(b) Modes $\tilde{p}_{x}-\tilde{m}_{1 x}$ and $\tilde{p}_{y}-\tilde{m}_{1 y}$.
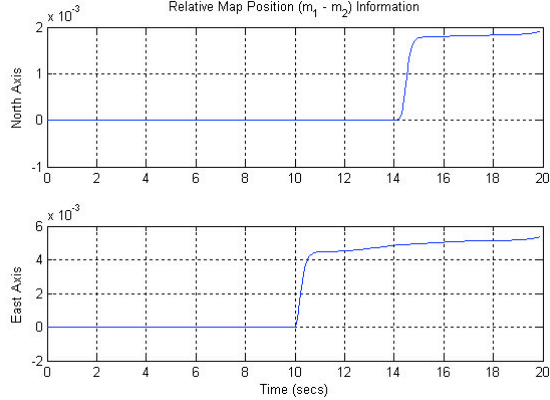

(c) Modes $\tilde{m}_{1 x}-\tilde{m}_{2 x}$ and $\tilde{m}_{1 y}-\tilde{m}_{2 y}$.

Fig. 4. Marginalised information of modes. a) These modes remain unobservable regardless of any maneuvering by the vehicle; b) these modes only become fully observable when the vehicle traverses laterally to each of the features; c) the easterly component (y-axis) becomes observable when the vehicle begins to move (after 10 seconds) but the northerly component (x-axis) only becomes fully observable when the vehicle traverses laterally to each of the features (after 14 seconds).

north, information about the relative position of the first feature w.r.t the second feature in the easterly direction starts to increase and it is only after the vehicle starts to move east that the information about the relative position of feature 1 w.r.t both the vehicle and feature 2 on both axes starts to increase as all of these states become observable.

\section{DisCUSSION AND CONCLUSIONS}

The purpose of this paper is to achieve a better understanding of bearing-only SLAM systems from a control theory viewpoint. Our analysis was focused on determining the bases for the nullspace of the observability matrices for our system. Linear observability analyses were performed by treating our system as piecewise linear and under the consideration of bearing-only measurements.

Specifically, the observability analysis indicated the instantaneous unobservable modes in the system and the directions in the state space for which no information is being added over the set of observations. Our results can be used to determine the the set of motions that the vehicle must take in order to result in maximum observability and thus induce constrained error drift in the estimation task.

An example application in which maneuvers for path segments are chosen so as to enhance observability conditions for a UAV is given in [7] for the case of range and bearing SLAM. An equivalent strategy could be developed using the theoretical results in this paper to the case of a planar vehicle with bearing only sensing.

For the planar vehicle, our analysis showed that the orientation is the only state that can be recovered in one step. In two steps, the sensor-centric model of the planar vehicle performing SLAM with bearing-only measurements is fully observable. We have formalised the notion that triangulation from different positions is needed to fully recover pose and have demonstrated the concept using tools from control theory. As a consequence to these findings, it is important to avoid the case of zero velocity for a planar vehicle.

An interesting conclusion of the paper is that the number of unobservable modes in the world centric case reaches a lower limit. Adding more time segments to the analysis of any world-centric model is also of little use to estimating the global position of the vehicle or features, as these modes will always be unobservable. The use of anchors or using a sensor-centric approach allows the system to become fully observable when the appropriate vehicle motions are made.

In future work, we will examine SLAM using non-linear observability analysis and compare the differences between the non-linear and TOM/SOM linearised analysis.

\section{ACKNOWLEDGEMENTS}

This work is supported in part by the EU URUS project FP6-IST-045062, the Spanish Ministry of Education and Science under a FPI Scholarship to TVC, a Ramón y Cajal Postdoctoral Fellowship to JAC and the project DPI 20045414 to AS, and by the ARC Centre of Excellence programme, funded by the Australian Research Council (ARC) and the New South Wales State Government to MB and SS.

\section{REFERENCES}

[1] G. G. P. Bonnifait, "Design and experimental validation of an odometric and goniometric localization system for outdoor robot vehicles," IEEE Trans. Robot. Automat., vol. 14, no. 4, Aug. 1998.

[2] R. S. A. Martinelli, "Observability analysis for mobile robot localization," in Proc. IEEE/RSJ Int. Conf. Intell. Robots Syst., Edmonton, Aug. 2005

[3] A. K. R. Hermann, "Nonlinear observability and controllability," IEEE Trans. Automat. Contr., vol. 22, no. 5, 1977.

[4] A. Bicchi, D. Pratichizzo, A. Marigo, and A. Balestrino, "On the observability of mobile vehicles localization," in Proc. 6th IEEE Mediterranenan Conf. Control Automat., Sardinia, Jun. 1998.

[5] D. Goshen-Meskin and I. Bar-Itzhack, "Observability analysis of piece-wise constant systems - Part I: Theory," IEEE Trans. Aerosp. Electron. Syst., vol. 28, no. 4, pp. 1056-1067, Oct. 1992.

[6] J. Kim and S. Sukkarieh, "Improving the real-time efficiency of inertial SLAM and understanding its observability," in Proc. IEEE/RSJ Int. Conf. Intell. Robots Syst., Sendei, Sep. 2004, pp. 21-26.

[7] M. Bryson and S. Sukkarieh, "Active airborne localisation and exploration in unknown environments using inertial SLAM," in Proc. IEEE Aerospace Conf., Big Sky, Mar. 2006.

[8] J. Andrade-Cetto and A. Sanfeliu, "The effects of partial observability when building fully correlated maps," IEEE Trans. Robot., vol. 21 , no. 4, pp. 771-777, Aug. 2005.

[9] P. S. Maybeck, Stochastic Models, Estimation, and Control. New York: Academic Press, 1979, vol. 1.

[10] R. Eustice, M. Walter, and J. J. Leonard, "Sparse extended information filters: Insights and sparsification," in Proc. IEEE/RSJ Int. Conf. Intell. Robots Syst., Edmonton, Aug. 2005. 